Edition 1985, Lea \& Febger, Philadelphia). Parenterally administered copper corrects the hepatic copper deficiency and restores serum copper and ceruloplasmin levels to normal but may not arrest the progressive cerebral degeneration. Nonetheless, Menkes advises initiation of copper therapy early since the clinical course of the disease is variable.

\title{
NEURO-OPHTHALMOLOGY
}

\section{TONIC UPGAZE IN INFANCY}

Tonic upward ocular deviation without seizure activity or neurologic disease is reported in 3 infants from the Pediatric and Neuro-ophthalmology Units, School of Medicine, University of California, San Francisco. The frequency and duration of the tonic upgaze episodes decreased with time but were exacerbated by fatigue or illness. Downward eye movements were normal. (Ahn JC et al. Tonic upgaze in infancy. A report of three cases. Arch Ophthalmol Jan 1989;107:57-58).

COMMENT. Transient tonic downward gaze in newborns is a fairly common phenomenon whereas tonic upgaze deviation is rarely reported, except with seizure activity, visual loss, or brain-stem disease. The pathophysiology of this transient variety of tonic upgaze is unknown but appears to differ from that of the downgaze type, being more persistent and exacerbated by fatigue or illness.

\section{OCULAR SIGNS OF CONGENITAL VARICELIAA}

Ocular abnormalities associated with congenital varicella are described in 3 children examined at the Hospital for Sick Children, Great Ormond Street, London, England. The syndrome followed a maternal varicella infection during the second trimester of pregnancy. Ocular findings included chorioretinitis, atrophy and hypoplasia of optic discs, cataract, and Horner's syndrome. Neurological abnormalities included bulbar palsy, hemiparesis, learning difficulties, and psychomotor retardation. The authors stress that the ocular lesions may be subtle and routine ophthalmological evaluation in infants with the congenital varicella syndrome is advisable. (Lambert SR, Taylor D et al. Ocular manifestations of the congenital varicella syndrome. Arch Ophthalmol Jan 1989;107: $52-56$ ).

COMMENT. Chorioretinal scarring occurs after intrauterine rubella, toxoplasmosis, syphilis, and less frequently with intrauterine herpes simplex and cytomegalovirus infections. Varicella chorioretinitis should be included in the differential diagnosis of congenital chorioretinal scarring. Optic nerve hypoplasia has not previously been reported in association with congenital varicella syndrome. 